
\title{
$\angle$ Research Square \\ Predictive Value of F-SIRI in Prognosis of Patients with Hepatocellular Carcinoma after Radical Hepatectomy
}

hu panyi

First Affiliated Hospital of Anhui Medical University https://orcid.org/0000-0002-9291-1391

\section{Yongwei Zhang}

First Affiliated Hospital of Anhui Medical University

yeben qian ( $\nabla$ qianyeben@hotmail.com )

First Affiliated Hospital of Anhui Medical University https://orcid.org/0000-0002-3145-3745

\section{Research}

Keywords: hepatocellular carcinoma, hepatectomy, fibrinogen, systemic inflammatory response index, prognosis

Posted Date: July 26th, 2021

DOI: https://doi.org/10.21203/rs.3.rs-651278/v1

License: (c) (i) This work is licensed under a Creative Commons Attribution 4.0 International License. Read Full License 


\section{Abstract}

Background: Objective to evaluate the predictive value of preoperative fibrinogen and systemic inflammatory response index (F-SIRI) in the prognosis of patients with hepatocellular carcinoma after radical hepatectomy.

Methods: the clinical data of 298 patients with hepatocellular carcinoma who underwent surgery and confirmed by postoperative pathology in our hospital from January 2015 to December 2017 were retrospectively analyzed. The F-SIRI score was calculated according to FIB and SIRI data of peripheral blood. The relationship between F-SIRI score and clinicopathological characteristics was analyzed. The survival analysis was performed by Kaplan Meier method, Cox regression analysis was used to analyze the prognostic factors.

Results: preoperative F-SIRI score was correlated with tumor diameter, FIB and SIRI $(P<0.05)$, but not with age, gender, TNM stage and other clinical features $(P>0.05)$. There were significant differences in the 5year DFS rate and OS rate among patients with different preoperative F-SIRI scores $(P<0.05)$; Cox regression analysis showed that preoperative tumor diameter, alpha fetoprotein level and F-SIRI score were independent predictors of DFS in patients with $\mathrm{HCC}(P<0.05)$, while preoperative tumor diameter, ALB and F-SIRI score as independent predictors of OS $(P<0.05)$.

Conclusion: preoperative F-SIRI is an independent prognostic factor in patients with HCC after radical hepatectomy, with poor prognosis in patients with high level of F-SIRI.

\section{Background}

According to GLOBOCAN 2018 data[1], there were 841080 new cases of liver cancer in the world in 2018, including 596574 male cases. There were 244506 cases of liver cancer in women. The incidence rate of global liver cancer is $11.0 / 10$ million, and the standardized incidence rate in the world is $9.3 / 10$ million. Liver cancer has become the sixth most common cancer in the world and the fourth leading cause of cancer death in the world and half of new cases of liver cancer occur in China every year. Therefore, we are actively looking for more effective treatment as lacking reliable prognostic indicators to guide clinical treatment decisions. Tumor - related inflammatory response, as an obvious feature of malignant tumor, plays an important role in the occurrence and development of malignant tumor[2]. Fibrinogen (FIB), a glycoprotein secreted by hepatocytes, as a key regulator of coagulation system, plays an important role in coagulation, cell adhesion and systemic inflammatory response. Increased fibrinogen level occurs in a variety of malignant tumors, including liver cancer, which is significantly correlated with tumor progression, invasion, distant metastasis and low survival rate [3, 4]. Peripheral blood cells, including neutrophils, lymphocytes and monocytes, are common indicators of inflammatory response. In addition, a variety of new inflammatory factors can be used to predict the prognosis of patients with malignant tumors, such as neutrophil / lymphocyte ratio (NLR), lymphocyte to monocyte ratio (LMR), as well as the new prediction model (F-NLR) established by FIB and NLR showing good prediction effect in the 
prognosis evaluation of patients with resectable gastric cancer [5]. In recent years, systemic inflammatory response index (SIRI) based on peripheral blood neutrophil, monocyte and lymphocyte counts has been considered as a predictor of the prognosis of liver cancer [6]. Therefore, we propose a prognostic index based on preoperative FIB and SIRI (F-SIRI) to explore whether it can accurately predict the survival and prognosis of patients with hepatocellular carcinoma.

\section{Subject And Method}

\subsection{Subject}

298 patients with hepatocellular carcinoma who were received by our hospital from January 2015 to December 2017 were selected, including 251 males and 47 females, with an age of (52.6 \pm 10.8$)$ years old. Inclusive criteria: (1) conducted radical hepatectomy; (2) confirmed hepatocellular carcinoma according to postoperative pathology; (3) The preoperative laboratory data included FIB and SIRI data; (4) The clinical and follow-up data were complete. Exclusion criteria: (1) patients received chemotherapy before operation; (2) Patients with severe acute and chronic infection; (3) Patients with severe hematological diseases; (4) Patients with other malignant tumors; (5) whose data missing.

\subsection{Observation Index and Definition of F-SIRI}

SIRI = neutrophil count $\times$ Monocyte count $/$ lymphocyte count. According to the instructions of the instrument, the normal range of plasma FIB is $2.0 \sim 4.0 \mathrm{~g} / \mathrm{L}$, which is defined as Hyper-FIBemia when higher than $4.0 \mathrm{~g} / \mathrm{L}$, so $4.0 \mathrm{~g} / \mathrm{L}$ as the preoperative FIB critical value [7]. The critical value of SIRI was 0.9 by using the method of maximum determined rank statistics, which is a high expression when higher than 0.9. Based on the expression level of FIB and SIRI, the F-SIRI score was calculated. The score standard: FIB $\leq 4.0 \mathrm{~g} / \mathrm{l}$ is 0 , and $>4.0 \mathrm{~g} / \mathrm{l}$ is 1 ; The score of $\mathrm{SIRI} \leq 0.9$ is 0 , and the score of $\mathrm{SIRI}>0.9$ is 1 ; The sum of FIB and SIRI scores is F-SIRI score.

\subsection{Follow-up}

The patients were followed up by outpatient and telephone, with every 3 months in the first two years and once every six months after the operation, till to December 2020 or the patient died, with the median follow-up time of $35(6-75)$ months. Overall survival (OS) time refers to the time from the beginning of the day after surgery to the end of the patient's death or follow-up. Disease free survival (DFS) is defined as the time from the day after surgery to the end of local recurrence / distant metastasis or follow-up.

\subsection{Statistical Methods}

SPSS20.0 software was used for statistical analysis. The count data were compared by $\chi^{2}$ test; The measurement data were expressed by the mean value \pm standard deviation $(x \pm s)$, the comparation was tested by t-test; survival curve was draw according to Kaplan-Meier method, and Log-rank test was 
performed for comparison between groups; Cox regression model was used for multivariate survival analysis. $P<0.05$ refers to significant difference.

\section{Results}

\subsection{Clinicopathological Characteristics}

Among the 298 patients, 235 (78.9\%) were younger than 60 years old and $63(21.1 \%)$ were older than 60 years old; 251 males (84.2\%) and 47 females (15.8\%); 170 cases (57.0\%) with cirrhosis, 128 (43.0\%) without cirrhosis; 125 cases $(41.9 \%)$ with tumor diameter $\leq 5 \mathrm{~cm}$ and 173 cases $(58.1 \%)$ with tumor diameter $>5 \mathrm{~cm} ; 229$ cases $(76.8 \%)$ of TNM stage I and 69 cases (23.2\%) of II IIla stage; Other clinicopathological characteristics are shown in Table 1. 
Table 1

The clinicopathological characteristics of patients and the relationship with preoperative F-SIRI [cases (\%)]

\begin{tabular}{|c|c|c|c|c|c|c|}
\hline \multirow{2}{*}{$\begin{array}{l}\text { Clinical } \\
\text { Characteristics }\end{array}$} & \multirow{2}{*}{$\begin{array}{l}\text { Number of } \\
\text { cases }\end{array}$} & \multicolumn{3}{|c|}{ F-SIRI(scores) } & \multirow{2}{*}{$\begin{array}{l}F / X^{2} \\
\text { value }\end{array}$} & \multirow{2}{*}{$\begin{array}{l}P \\
\text { value }\end{array}$} \\
\hline & & 0 & 1 & 2 & & \\
\hline Age (years) & & & & & 0.301 & 0.860 \\
\hline$<60$ & 235(78.9) & $121(80.1)$ & $65(77.4)$ & 47(77.8) & & \\
\hline$\geq 60$ & $63(21.1)$ & $30(19.9)$ & 17(22.6) & $16(22.2)$ & & \\
\hline Gender & & & & & 2.736 & 0.268 \\
\hline male & $251(84.2)$ & 117(77.5) & 61(72.6) & $53(84.1)$ & & \\
\hline female & $47(15.8)$ & $34(22.5)$ & $23(27.4)$ & 10(15.9) & & \\
\hline cirrhosis & & & & & 0.192 & 0.909 \\
\hline yes & $170(57.0)$ & $88(58.3)$ & $47(56.0)$ & $35(55.6)$ & & \\
\hline no & $128(43.0)$ & $63(41.7)$ & $37(44.1)$ & $28(44.4)$ & & \\
\hline Tumor diameter(cm) & & & & & 13.857 & 0.001 \\
\hline$\leq 5$ & $125(41.9)$ & $79(52.3)$ & 28(33.3) & 18(28.6) & & \\
\hline$>5$ & $173(58.1)$ & $72(47.8)$ & $56(66.7)$ & 45(71.4) & & \\
\hline $\begin{array}{l}\text { Microvascular } \\
\text { invasion }\end{array}$ & & & & & 2.933 & 0.231 \\
\hline yes & $80(26.8)$ & $34(22.5)$ & $26(31.0)$ & 20(31.8) & & \\
\hline no & $218(73.2)$ & $117(77.5)$ & $58(69.0)$ & $43(68.2)$ & & \\
\hline TNM staging & & & & & 2.947 & 0.229 \\
\hline I stage & $229(76.8)$ & $111(73.5)$ & 70(83.3) & 48(76.2) & & \\
\hline II IIla stage & $69(23.2)$ & $40(26.5)$ & 14(16.7) & 15(23.8) & & \\
\hline HBsAg positive & & & & & 2.879 & 0.237 \\
\hline yes & $256(85.9)$ & $131(86.8)$ & $68(80.1)$ & $57(90.5)$ & & \\
\hline no & $42(14.1)$ & $20(13.2)$ & 16(19.1) & $6(9.5)$ & & \\
\hline $\operatorname{ALB}(\mathrm{g} / \mathrm{L})$ & & & & & 0.741 & 0.691 \\
\hline$\leq 35$ & $163(54.7)$ & $86(57.0)$ & $43(51.2)$ & $34(54.0)$ & & \\
\hline$>35$ & $135(45.3)$ & $65(43.0)$ & $41(48.8)$ & $29(46.0)$ & & \\
\hline AST(U/L) & & & & & 1.323 & 0.516 \\
\hline
\end{tabular}




\begin{tabular}{|c|c|c|c|c|c|c|}
\hline \multirow{2}{*}{$\begin{array}{l}\text { Clinical } \\
\text { Characteristics }\end{array}$} & \multirow{2}{*}{$\begin{array}{l}\text { Number of } \\
\text { cases }\end{array}$} & \multicolumn{3}{|c|}{ F-SIRI(scores) } & \multirow{2}{*}{$\begin{array}{l}F / \chi^{2} \\
\text { value }\end{array}$} & \multirow{2}{*}{$\begin{array}{l}\mathrm{P} \\
\text { value }\end{array}$} \\
\hline & & 0 & 1 & 2 & & \\
\hline$\leq 40$ & $161(54.0)$ & $84(55.6)$ & $47(56.0)$ & $30(47.6)$ & & \\
\hline$>40$ & $137(46.0)$ & $67(44.4)$ & $37(44.0)$ & $33(52.4)$ & & \\
\hline $\operatorname{ALT}(\mathrm{U} / \mathrm{L})$ & & & & & 1.532 & 0.465 \\
\hline$\leq 40$ & $170(57.0)$ & $82(54.3)$ & $48(57.1)$ & $40(63.5)$ & & \\
\hline$>40$ & $128(43.0)$ & $69(45.7)$ & $36(42.9)$ & $23(36.5)$ & & \\
\hline $\begin{array}{l}\text { Alpha- } \\
\text { fetoprotein }(\mu \mathrm{g} / \mathrm{L})\end{array}$ & & & & & 0.183 & 0.913 \\
\hline$\leq 400$ & $169(56.7)$ & $84(55.6)$ & $48(57.1)$ & $37(58.7)$ & & \\
\hline$>400$ & $129(43.3)$ & $67(44.4)$ & $36(42.9)$ & $26(41.3)$ & & \\
\hline SIRI & & $\begin{array}{l}0.72 \pm \\
0.25\end{array}$ & $\begin{array}{l}1.32 \pm \\
0.34\end{array}$ & $\begin{array}{l}1.69 \pm \\
0.58\end{array}$ & 12.871 & $\begin{array}{l}<.001 \\
0.0\end{array}$ \\
\hline $\mathrm{FIB}(\mathrm{g} / \mathrm{L})$ & & $\begin{array}{l}3.11 \pm \\
0.42\end{array}$ & $\begin{array}{l}4.08 \pm \\
0.86\end{array}$ & $\begin{array}{l}4.59 \pm \\
0.92\end{array}$ & 14.121 & $\hat{0.001}$ \\
\hline
\end{tabular}

2.2 Relationship between preoperative F-SIRI and clinicopathological characteristics of hepatocellular carcinoma patients

Of the 298 patients, $151(50.7 \%)$ had a preoperative F-SIRI score of 0, $84(28.2 \%)$ had a preoperative FSIRI score of 1 , and $63(21.1 \%)$ had a preoperative F-SIRI score of 2 . Univariate analysis showed that preoperative F-SIRI score was correlated with tumor diameter, SIRI and FIB $(P<0.05)$, but not with age, gender, TNM stage and other clinical features $(P>0.05)$. See Table 1.

2.3 Effect of preoperative F-SIRI on prognosis of patients with hepatocellular carcinoma

There were significant differences in DFS and OS among patients with different preoperative F-SIRI scores $\left(X^{2}=57.375,29.105\right.$, $P$ value $\left.<0.001\right)$; The 5-year DFS rate and OS rate were $61.4 \%$ and $69.2 \%$ respectively in patients with F-SIRI score 0; The 5-year DFS rate and OS rate were $45.6 \%$ and $54.6 \%$ respectively in patients with F-SIRI score 1 ; The 5 -year DFS rate was $26.7 \%$ and OS rate was $38.5 \%$ in patients with F-SIRI score of 2.

Cox regression analysis indicated that preoperative tumor diameter, alpha fetoprotein level and F-SIRI score were independent predictors of DFS in patients with HCC $(P<0.05)$, while preoperative tumor diameter, ALB and F-SIRI score were independent predictors of OS $(P<0.05)$. 
Table 2

Univariate and Multivariate Analysis on Influencing Factors of DFS in 298 Patients with Hepatocellular Carcinoma

\begin{tabular}{|c|c|c|c|c|c|c|}
\hline \multirow{2}{*}{$\begin{array}{l}\text { Clinicopathological } \\
\text { characteristics }\end{array}$} & \multicolumn{3}{|c|}{ Simple Correlation } & \multicolumn{3}{|c|}{ Multivariate Analysis } \\
\hline & $\beta$ & $H R(95 \% \mathrm{Cl})$ & $\begin{array}{l}P \\
\text { value }\end{array}$ & $\beta$ & $H R(95 \% \mathrm{Cl})$ & $\begin{array}{l}P \\
\text { value }\end{array}$ \\
\hline Age & 0.239 & $\begin{array}{l}1.241(0.978 \sim \\
1.296)\end{array}$ & 0.627 & - & - & - \\
\hline Gender & 0.329 & $\begin{array}{l}1.014(0.921 \sim ~ \\
1.465)\end{array}$ & 0.728 & - & - & - \\
\hline Cirrhosis & 0.351 & $\begin{array}{l}1.021(0.896 \sim \\
1.074)\end{array}$ & 0.711 & - & - & - \\
\hline Tumor Diameter & 0.512 & $\begin{array}{l}1.541(1.175 \sim \\
1.871)\end{array}$ & $\hat{0} .001$ & 0.571 & $\begin{array}{l}1.456(1.212 \sim \\
1.768)\end{array}$ & $\begin{array}{l}< \\
0.001\end{array}$ \\
\hline Microvascular invasion & 0.478 & $\begin{array}{l}1.436(1.087 \sim \\
1.747)\end{array}$ & 0.039 & 0.492 & $\begin{array}{l}1.412(1.187 \sim \\
1.698)\end{array}$ & 0.053 \\
\hline TNM staging & 0.378 & $\begin{array}{l}1.215(1.017 \sim ~ \\
1.487)\end{array}$ & 0.316 & - & - & - \\
\hline $\begin{array}{l}\text { HBsAg } \\
\text { positive }\end{array}$ & 0.376 & $\begin{array}{l}1.325(0.858 \sim ~ \\
1.478)\end{array}$ & 0.412 & - & - & - \\
\hline ALB & -0.036 & $\begin{array}{l}0.559(0.368 \sim ~ \\
2.826)\end{array}$ & 0.005 & -0.041 & $\begin{array}{l}0.612(0.411 \sim \\
0.745)\end{array}$ & 0.079 \\
\hline AST & 0.312 & $\begin{array}{l}1.158(0.876 \sim \\
1.321)\end{array}$ & 0.059 & - & - & - \\
\hline ALT & 0.298 & $\begin{array}{l}1.041(0.912 \sim \\
1.345)\end{array}$ & 0.061 & - & - & - \\
\hline Alpha-fetoprotein & 0.325 & $\begin{array}{l}1.176(0.875 \sim ~ \\
1.274)\end{array}$ & 0.017 & 0.339 & $\begin{array}{l}1.058(0.912 \sim \\
1.313)\end{array}$ & 0.025 \\
\hline $\begin{array}{l}\text { F-SIRI } \\
\text { score }\end{array}$ & 0.574 & $\begin{array}{l}1.551(1.134 \sim \\
1.796)\end{array}$ & $\hat{0} .001$ & 0.492 & $\begin{array}{l}1.478(1.126 \sim \\
1.737)\end{array}$ & $<.001$ \\
\hline
\end{tabular}


Table 3

Univariate and Multivariate Analysis on Influencing Factors of OS in 298 Patients with Hepatocellular Carcinoma

\begin{tabular}{|c|c|c|c|c|c|c|}
\hline \multirow{2}{*}{$\begin{array}{l}\text { Clinicopathological } \\
\text { characteristics }\end{array}$} & \multicolumn{3}{|c|}{ Simple Correlation } & \multicolumn{3}{|c|}{ Multivariate Analysis } \\
\hline & $\beta$ & $H R(95 \% \mathrm{Cl})$ & $\begin{array}{l}P \\
\text { value }\end{array}$ & $\beta$ & $H R(95 \% \mathrm{Cl})$ & $\begin{array}{l}P \\
\text { value }\end{array}$ \\
\hline Age & 0.223 & $\begin{array}{l}1.089(0.876 \sim \\
1.312)\end{array}$ & 0.587 & - & - & - \\
\hline Gender & 0.229 & $\begin{array}{l}1.121(0.964 \sim ~ \\
1.389)\end{array}$ & 0.676 & - & - & - \\
\hline Cirrhosis & 0.312 & $\begin{array}{l}1.211(0.912 \sim \\
1.369)\end{array}$ & 0.598 & - & - & - \\
\hline Tumor Diameter & 0.474 & $\begin{array}{l}1.376(1.115 \sim \\
1.578)\end{array}$ & $\hat{0} .001$ & 0.512 & $\begin{array}{l}1.422(1.111 \sim \\
1.768)\end{array}$ & $\begin{array}{l}<.001 \\
0.01\end{array}$ \\
\hline Microvascular invasion & 0.436 & $\begin{array}{l}1.312(1.058 \sim \\
1.425)\end{array}$ & 0.043 & 0.471 & $\begin{array}{l}1.389(1.077 \sim \\
1.628)\end{array}$ & 0.062 \\
\hline TNM staging & 0.322 & $\begin{array}{l}1.236(1.121 \sim \\
1.369)\end{array}$ & 0.286 & - & - & - \\
\hline $\begin{array}{l}\text { HBsAg } \\
\text { positive }\end{array}$ & 0.364 & $\begin{array}{l}1.278(0.923 \sim \\
1.521)\end{array}$ & 0.396 & - & - & - \\
\hline ALB & -0.049 & $\begin{array}{l}0.662(0.368 \sim ~ \\
2.826)\end{array}$ & 0.009 & -0.052 & $\begin{array}{l}0.712(0.514 \sim \\
0.946)\end{array}$ & 0.023 \\
\hline AST & 0.266 & $\begin{array}{l}1.058(0.849 \sim \\
1.158)\end{array}$ & 0.063 & - & - & - \\
\hline ALT & 0.323 & $\begin{array}{l}1.121(0.896 \sim \\
1.246)\end{array}$ & 0.058 & - & - & - \\
\hline Alpha-fetoprotein & 0.314 & $\begin{array}{l}1.254(0.912 \sim \\
1.343)\end{array}$ & 0.022 & 0.296 & $\begin{array}{l}1.125(0.936 \sim \\
1.276)\end{array}$ & 0.053 \\
\hline $\begin{array}{l}\text { F-SIRI } \\
\text { score }\end{array}$ & 0.612 & $\begin{array}{l}1.476(1.296 \sim \\
1.713)\end{array}$ & $\hat{0} .001$ & 0.589 & $\begin{array}{l}1.397(1.096 \sim \\
1.512)\end{array}$ & $\langle .001$ \\
\hline
\end{tabular}

\section{Discussion}

The evaluation of study on the prognostic significance of preoperative F-SIRI in patients with hepatocellular carcinoma showed that preoperative F-SIRI was independently related to the prognosis of patients, with higher risk of recurrence / metastasis and poorer prognosis in patients with higher preoperative F-SIRI level. F-SIRI, a new parameter established by combining FIB and peripheral blood 
neutrophil, monocyte and lymphocyte counts, is yet not reported its application in prognosis evaluation in China.

There is a consensus that cancer-related inflammation is an important marker of cancer[8-10]. As more and more evidence showed, local and systemic inflammatory response, plays an important role in promoting tumor growth, deterioration and metastasis, is closely related to the long-term prognosis of patients. Neutrophils can down-regulate the immune function of host cells against cancer cells, thus play an important role in inflammatory response; As the key factor of anti-tumor immunity, lymphocytes participated in the humoral cellular immune process can inhibit the proliferation and metastasis of tumor; while the tumor associated macrophages produced by monocytes can secrete a variety of cytokines such as metalloproteinases after the stimulation of chemokines to the tumor, so play a role in promoting angiogenesis. SIRI, as a new inflammatory index proposed in recent years, is calculated by the counts of neutrophils, monocytes and lymphocytes in peripheral blood. SIRI as an independent predictor of postoperative recurrence and survival in patients with pancreatic cancer, Qi et al. [11] first proposed, it can be more predictive than NLR and LMR. Liu et al. [12] evaluated the application value of SIRI in the prognosis of patients undergoing radical gastrectomy. The results show that as an independent influencing factor of DFS and OS, SIRI is closely related to tumor size, TNM stage and lymphatic invasion. Xu et al. [6]suggested that preoperative SIRI is a reliable predictor of postoperative survival in patients with liver cancer. Therefore, SIRI can also predict the prognosis of tumor.

Other studies also said that hypercoagulable state of blood is related to the occurrence and development of malignant tumor $[13,14]$. Abnormal hypercoagulable state of blood can promote the proliferation and invasion of tumor to help the immune escape of tumor. FIB, as a typical coagulation factor, plays an important role in the process of coagulation. Firstly, besides hepatocytes, it can also be synthesized endogenous by cancer cells[15]. Then, fibrinogen can be directly combined with certain growth factors, such as transforming growth factor-B (TGF-B), fibroblast growth factor (FGF), vascular endothelial growth factor (VEGF), and platelet-derived growth factor (PDGF) family to regulate the growth of cancer cells[15, 16]. Also, it is believed that inflammation is involved in the progression of tumor, and fibrinogen is a kind of p-globulin which belongs to pro-inflammatory protein and may lead to the elevation of various inflammatory cytokines $[17,18]$. Next, increased fibrinogen can enhance cell migration and invasion by regulating the expression of vimentin and cadherin, and by inducing epithelial mesenchymal transition (EMT)[19]. In addition, fibrin helps platelets adhere to tumor cells, while platelets promote more fibrin to gather around tumor cells by forming thrombin. They are promoting each other to protect tumor cells from natural killer cell toxicity. Even tumor cells prefer to adhere to fibrinogen rather than platelets[20]. $[21,22]$ It has been recognized that high levels of fibrinogen receptors are expressed in some tumor cells, including intercellular adhesion molecule 1 (ICAM-1) and $a 5, \beta 1$, $a 3$, and $\beta 3$ integrator. Therefore, fibrinogen promotes the bridge connection between platelets and circulating tumor cells (CTC), helping platelets adhere to tumor cells. With the formation of this matrix barrier, carbon tetrachloride escapes from the natural killer cell-induced elimination. It promotes cell migration by providing a matrix for tumor cell migration and by interacting with adhesion molecules and integrins. Fibrinogen can also mediate endothelial cell adhesion to carbon tetrachloride through ICAM-1. On the other hand, Palumbo et al. 
(2000)[23] proved that the metastatic potential of circulating tumor cells depends to a large extent on fibrinogen, and showed a therapeutic strategy focusing on hemostatic factors to control solid tumor metastasis.

On the other hand, some study [24] found that increased FIB involves with poor prognosis of gastric cancer patients. Yu et al. [25] indicated that Hyper FIBemia is closely related to tumor progression as an independent factor for poor prognosis of cancer patients. Li et al.[26] showed that increased FIB in peripheral blood is an independent risk factor for survival and prognosis of patients with multiple myeloma. These results suggest that FIB may be a predictor of tumor prognosis.

Based on previous studies, we propose a new parameter F-SIRI, which combined FIB and SIRI to better predict the prognosis of HCC patients. This study shows that F-SIRI is correlated with tumor diameter, but not with TNM stage, which may be that lymph node metastasis mainly reflects the aggressive behavior of tumor, but not inflammatory response, therefore requires further research. Survival analysis showed that F-SIRI was associated with DFS and OS in HCC patients. The 5-year DFS and OS rates of patients with higher F-SIRI level were lower; in addition, Cox regression analysis indicated that F-SIRI was an independent predictor of DFS and OS in HCC patients, which was consistent with Gao et al. [27]. Therefore, F-SIRI as a new indicator of inflammatory response can be used to predict the prognosis of patients with hepatocellular carcinoma.

Based on previous studies, we propose a new parameter F-SIRI, which combined FIB and SIRI to better predict the prognosis of HCC patients. This study shows that F-SIRI is correlated with tumor diameter, but not with TNM stage, which may be that lymph node metastasis mainly reflects the aggressive behavior of tumor, but not inflammatory response, therefore requires further research. Survival analysis showed that F-SIRI was associated with DFS and OS in HCC patients. The 5-year DFS and OS rates of patients with higher F-SIRI level were lower; in addition, Cox regression analysis indicated that F-SIRI was an independent predictor of DFS and OS in HCC patients, which was consistent with Gao et al. [18]. Therefore, F-SIRI as a new indicator of inflammatory response can be used to predict the prognosis of patients with hepatocellular carcinoma.

Some limitations in this study: (1) the median follow-up time of 35 months is not enough to fully accumulate the number of deaths, so inevitably affect the stability of the results;(2) Cox regression model did not collect and control all confounding factors although the collected confounding factors were corrected as far as possible;(3) The study only preliminarily discuss the prognostic value of F-SIRI for HCC patients, but not compare with FIB, NLR and SIRI, thus needs further study.

\section{Conclusion}

In conclusion, this study preliminarily shows that F-SIRI can be used to predict prognosis as an independent influencing factor for HCC patients after radical hepatectomy. 


\section{Abbreviations}

F-SIRI: Fibrinogen and Systemic Inflammation Response Index

Fib: Fibrinogen

SIRI: Systemic Inflammation Response Index

TNM: Tumor Node Metastasis

DFS: Disease-free Survival

OS: Overall Survival

NLR: Neutrophil-to-Lymphocyte Ratio

LMR: Lymphocyte-Monocyte Ratio

AST: Alanine aminotransferase

ALB: Albumin

ALT: Alanine aminotransferase

\section{Declarations}

\section{Authors' contributions:}

Panyi Hu is responsible for writing and submitting the papers. Yongwei Zhang is responsible for data analysis and collation. Yeben Qian is responsible for the manuscript fees and ideas guidance. The authors read and approved the final manuscript.

\section{Funding:}

No.

\section{Availability of data and materials:}

All the initial data are from the medical record room of the First Affiliated Hospital of Anhui Medical University, and the data are true and valid.

\section{Ethics approval and consent to participate:}

The study was approved by the clinical research ethics committee of the First Affiliated Hospital of Anhui Medical University 


\section{Consent for publication:}

All data in this manuscript were from the medical record room of the First Affiliated Hospital of Anhui Medical University. All patients had obtained written informed consent before operation, and there was no detailed information of personal report in the manuscript.

\section{Competing interests:}

The authors declare that there is no conflict of interests.

\section{Acknowledgement:}

Thanks to the Natural Science Research Foundation of Anhui Province for supporting this research.

\section{References}

1. Bray F, Ferlay J, Soerjomataram I, Siegel RL, Torre LA, Jemal A. Global cancer statistics 2018: GLOBOCAN estimates of incidence and mortality worldwide for 36 cancers in 185 countries. Cancer J Clin. 2018;68(6):394-424.

2. Cortes E, Lachowski D, Rice A, et al. Retinoic Acid Receptor- $\beta$ Is Downregulated in Hepatocellular Carcinoma and Cirrhosis and Its Expression Inhibits Myosin-Driven Activation and Durotaxis in Hepatic Stellate Cells. Hepatology. 2019;69(2):785-802.

3. Xu WY, Zhang HH, Xiong JP, et al. Prognostic significance of the fibrinogen-to-albumin ratio in gallbladder cancer patients. World journal of gastroenterology. 2018;24(29):3281-92.

4. Zhang L, Chen QG, Li SQ, et al. Preoperative fibrinogen to prealbumin ratio as a novel predictor for clinical outcome of hepatocellular carcinoma. Future oncology (London England). 2019;15(1):1322.

5. Yamamoto M, Kurokawa Y, Kobayashi N, et al. Prognostic Value of the Combined Index of Plasma Fibrinogen and the Neutrophil-Lymphocyte Ratio in Gastric Cancer. World journal of surgery. 2020;44(1):207-12.

6. Xu L, Yu S, Zhuang L, et al. Systemic inflammation response index (SIRI) predicts prognosis in hepatocellular carcinoma patients. Oncotarget. 2017;8(21):34954-60.

7. Wakatsuki K, Matsumoto S, Migita K, et al. Preoperative Plasma Fibrinogen is Associated with Lymph Node Metastasis and Predicts Prognosis in Resectable Esophageal Cancer. World journal of surgery. 2017;41(8):2068-77.

8. Sun B, Karin M. Obesity, inflammation, and liver cancer. J Hepatol. 2012;56(3):704-13.

9. Tan Z, Xue H, Sun Y, Zhang C, Song Y, Qi Y. The Role of Tumor Inflammatory Microenvironment in Lung Cancer. Front Pharmacol. 2021;12:688625. 
10. Piñeiro Fernández J, Luddy KA, Harmon C, O'farrelly C. Hepatic Tumor Microenvironments and Effects on NK Cell Phenotype and Function. International journal of molecular sciences 20(17), (2019).

11. Qi Q, Zhuang $L$, Shen Y, et al. A novel systemic inflammation response index (SIRI) for predicting the survival of patients with pancreatic cancer after chemotherapy. Cancer. 2016;122(14):2158-67.

12. Liu Z, Ge H, Miao Z, Shao S, Shi H, Dong C. Dynamic Changes in the Systemic Inflammation Response Index Predict the Outcome of Resectable Gastric Cancer Patients. Frontiers in oncology. 2021;11:577043.

13. Im JH, Fu W, Wang $\mathrm{H}$, et al. Coagulation facilitates tumor cell spreading in the pulmonary vasculature during early metastatic colony formation. Cancer Res. 2004;64(23):8613-9.

14. Seth R, Tai LH, Falls T, et al. Surgical stress promotes the development of cancer metastases by a coagulation-dependent mechanism involving natural killer cells in a murine model. Ann Surg. 2013;258(1):158-68.

15. Sahni A, Simpson-Haidaris PJ, Sahni SK, Vaday GG, Francis CW. Fibrinogen synthesized by cancer cells augments the proliferative effect of fibroblast growth factor-2 (FGF-2). J Thromb Haemost. 2008;6(1):176-83.

16. Witsch E, Sela M, Yarden Y. Roles for growth factors in cancer progression. Physiology (Bethesda). 2010;25(2):85-101.

17. Shrihari TG. Dual role of inflammatory mediators in cancer. Ecancermedicalscience. 2017;11:721.

18. Musolino C, Allegra A, Pioggia G, Gangemi S. Immature myeloid-derived suppressor cells: A bridge between inflammation and cancer (Review). Oncol Rep. 2017;37(2):671-83.

19. Shu YJ, Weng H, Bao RF, et al. Clinical and prognostic significance of preoperative plasma hyperfibrinogenemia in gallbladder cancer patients following surgical resection: a retrospective and in vitro study. BMC Cancer. 2014;14:566.

20. Zheng S, Shen J, Jiao Y, et al. Platelets and fibrinogen facilitate each other in protecting tumor cells from natural killer cytotoxicity. Cancer Sci. 2009;100(5):859-65.

21. Steinbrecher KA, Horowitz NA, Blevins EA, et al. Colitis-associated cancer is dependent on the interplay between the hemostatic and inflammatory systems and supported by integrin alpha(M)beta(2) engagement of fibrinogen. Cancer Res. 2010;70(7):2634-43.

22. Roche $Y$, Pasquier D, Rambeaud JJ, Seigneurin D, Duperray A. Fibrinogen mediates bladder cancer cell migration in an ICAM-1-dependent pathway. Thromb Haemost. 2003;89(6):1089-97.

23. Palumbo JS, Kombrinck KW, Drew AF, et al. Fibrinogen is an important determinant of the metastatic potential of circulating tumor cells. Blood. 2000;96(10):3302-9.

24. Palaj J, Kečkéš Š, Marek V, Dyttert D, Waczulíková I, Durdík Š. Fibrinogen Levels Are Associated with Lymph Node Involvement and Overall Survival in Gastric Cancer Patients. Anticancer research. 2018;38(2):1097-104. 
25. Yu X, Hu F, Yao Q, Li C, Zhang H, Xue Y. Serum fibrinogen levels are positively correlated with advanced tumor stage and poor survival in patients with gastric cancer undergoing gastrectomy: a large cohort retrospective study. BMC Cancer. 2016;16:480.

26. Li QF, Zhang QK, Wei XF, Feng YF. [Coagulation Indexes and Their Prognostic Significance in Patients with Multiple Myeloma]. Zhongguo shi yan xue ye xue za zhi. 2021;29(3):791-6.

27. Gao W, Zhang F, Ma T, Hao J. High Preoperative Fibrinogen and Systemic Inflammation Response Index (F-SIRI) Predict Unfavorable Survival of Resectable Gastric Cancer Patients. J Gastric Cancer. 2020;20(2):202-11.

\section{Figures}



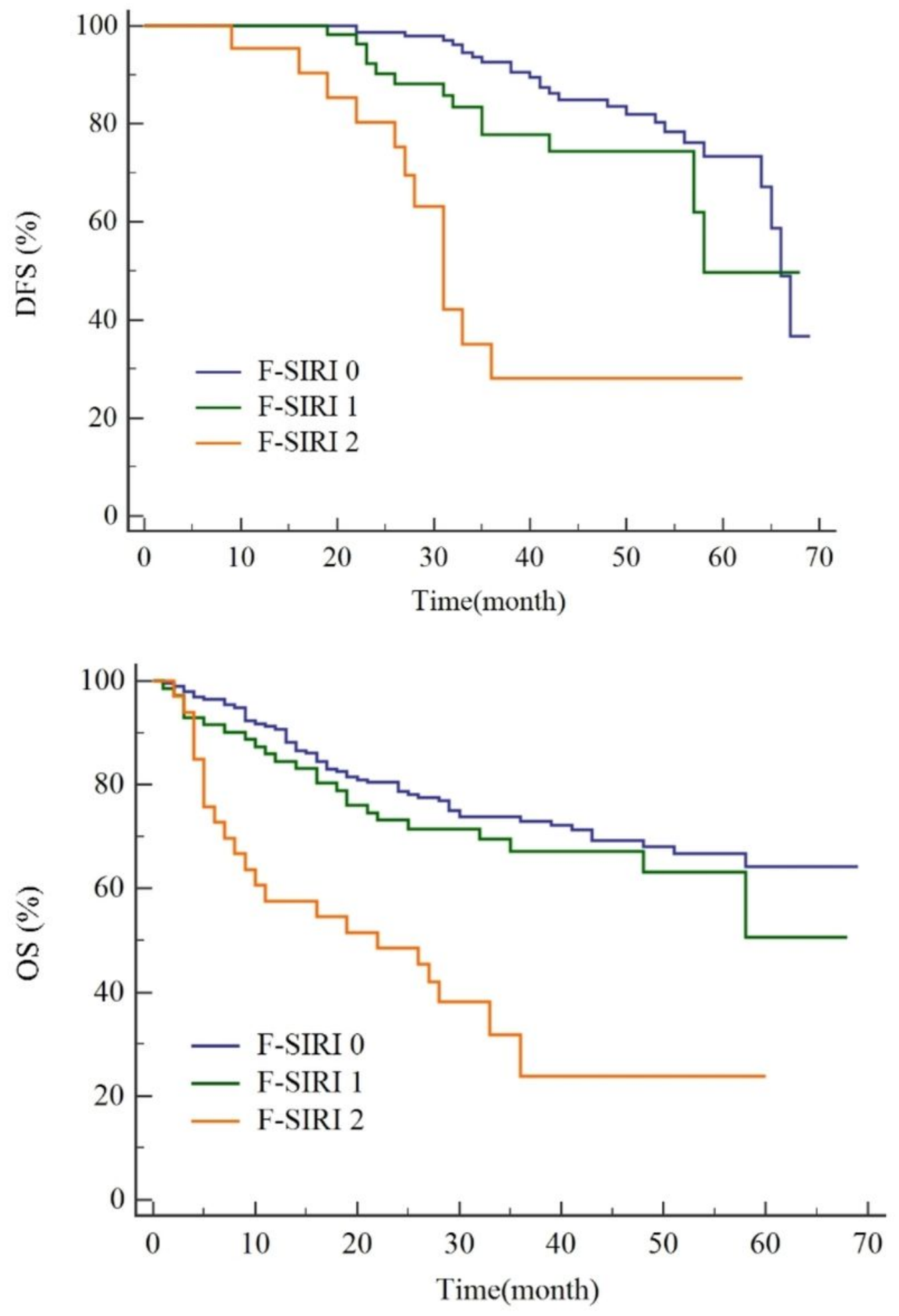

Figure 1

The Survival Curve of Patients with Different F-SIRI Scores 